\title{
Cross Cultural Adaptation, Validity, and Reliability of the Farsi Breastfeeding Attrition Prediction Tools in Iranian Pregnant Women
}

\author{
Forough Mortazavi ${ }^{1}$; Seyed Abbas Mousavi ${ }^{2, ;}$; Reza Chaman ${ }^{3}$; Ahmad Khosravi ${ }^{4}$; jill R. \\ Janke $^{5}$ \\ ${ }^{1}$ Department of Midwifery, Faculty of Nursing and Midwifery, Sabzevar University of Medical Sciences, Sabzevar, IR Iran \\ ${ }_{3}^{2}$ Research Center of Psychiatry, Golestan University of Medical Sciences, Gorgan, IR Iran \\ ${ }_{4}^{3}$ Department of Community Medicine, School of Medicine, Yasuj University of Medical Sciences, Yasuj, IR Iran \\ ${ }_{5}^{4}$ Center for Health Related Social and Behavioral Sciences Research, Shahroud University of Medical Sciences, Shahroud, IR Iran \\ ${ }^{5}$ Center for Health Related Social and Behavioral Sciences
Schorsing, University of Alaska, Anchorage, USA \\ *Corresponding Author: Seyed Abbas Mousavi, Research Center of Psychiatry, Golestan University of Medical Sciences, Gorgan, IR Iran. Tel: +98-2332395003, Fax: +98-2332395006, \\ E-mail: mosavi19@yahoo.com
}

Received: December 30, 2014; Revised: January 18, 2015; Accepted: February 15, 2015

\begin{abstract}
Background: The rate of exclusive breastfeeding in Iran is decreasing. The breastfeeding attrition prediction tools (BAPT) have been validated and used in predicting premature weaning.

Objectives:We aimed to translate the BAPT into Farsi, assess its content validity, and examine its reliability and validity to identify exclusive breastfeeding discontinuation in Iran.

Materials and Methods: The BAPT was translated into Farsi and the content validity of the Farsi version of the BAPT was assessed. It was administered to 356 pregnant women in the third trimester of pregnancy, who were residents of a city in northeast of Iran. The structural integrity of the four-factor model was assessed in confirmatory factor analysis (CFA) and exploratory factor analysis (EFA). Reliability was assessed using Cronbach's alpha coefficient and item-subscale correlations. Validity was assessed using the known-group comparison (128 with vs. 228 without breastfeeding experience) and predictive validity ( 80 successes vs. 265 failures in exclusive breastfeeding).

Results: The internal consistency of the whole instrument ( 49 items) was 0.775 . CFA provided an acceptable fit to the a priori four-factor model (Chi-square/df = 1.8, Root Mean Square Error of Approximation (RMSEA) $=0.049$, Standardized Root Mean Square Residual (SRMR) $=0.064$, Comparative Fit Index $(\mathrm{CFI})=0.911)$. The difference in means of breastfeeding control $(\mathrm{BFC})$ between the participants with and without breastfeeding experience was significant $(\mathrm{P}<0.001)$. In addition, the total score of BAPT and the score of Breast Feeding Control $(\mathrm{BFC})$ subscale were higher in women who were on exclusive breastfeeding than women who were not, at four months postpartum $(\mathrm{P}<$ 0.05).

Conclusions: This study validated the Farsi version of BAPT. It is useful for researchers who want to use it in Iran to identify women at higher risks of Exclusive Breast Feeding (EBF) discontinuation.
\end{abstract}

Keywords:Breast feeding; Validation Studies; Pregnant Women

\section{Background}

Breastfeeding has been known as the best way of infant feeding. It brings a number of health benefits for both mothers and their babies. The benefits of breastfeeding and the probability of a longer duration of breastfeeding increase when the baby is fed with breast milk exclusively $(1,2)$. The World Health Organization has recommended exclusive breastfeeding for the first six months of infant life (3).

The Iranian government successfully started promoting breastfeeding through a policy change by establishing the National Committee of Breastfeeding Promotion at the Ministry of Health in 1991 (4). The integrated monitoring evaluation system in the Family Health Office of the Ministry of Health conducted a retrospective study in 2007 on 63071 infants less than 24 months of age in all the 30 urban and rural provinces of Iran. The results of the study indicated that the rate of breastfeeding in the country at one and two years of age were $90 \%$ and 57\%, respectively (5). However, the rate of exclusive breastfeeding (EBF) at sixth months decreased from $44 \%$ in 2000 to $23 \%$ in 2010 (6). Results of a recent study in rural areas of northern Iran indicated that EBF duration of at least five months was $14.6 \%$ (7).

Previous studies have reported that the mother's perception of having insufficient milk or her perception of insufficient infant weight gain (8), low confidence (9), low breastfeeding self-efficacy (10), low breastfeeding knowledge (11), and receiving recommendations from pediatricians, family and friends $(12,13)$, were the reasons for the discontinuation of EBF. On the other hand, results of a systematic review showed that antepartum breastfeeding education and counseling were associated with increased EBF duration $(14,15)$. It is therefore necessary that women who are at risk of exclusive breastfeeding

Copyright (C) 2015, Iranian Red Crescent Medical Journal. This is an open-access article distributed under the terms of the Creative Commons Attribution-NonCommercial 4.0 International License (http://creativecommons.org/licenses/by-nc/4.0/) which permits copy and redistribute the material just in noncommercial usages, provided the original work is properly cited. 
discontinuation routinely be screened during pregnancy and proper intervention be conducted during the antenatal period. For this purpose, it is important to develop an appropriate instrument for the task of predicting EBF discontinuation in the antenatal period. Since breastfeeding is a behavior, an appropriate instrument must be developed based on a theory which can explain the breastfeeding behavior. The theory of planned behavior (TPB) explains and predicted breastfeeding (16-18). TPB constructs in breastfeeding are attitude, subjective norms, and perceived behavioral control towards breastfeeding. Attitude towards breastfeeding comprises two factors: women's positive or negative judgment of breastfeeding and evaluation of the outcomes of it for them and their babies. Subjective norms depend on women's perception about the significant others' judgment of breastfeeding and if they care about their opinions. Perceived behavioral control toward breastfeeding is women's perception of the degree of ease or difficulty of breastfeeding. The TPB assumptions in breastfeeding are: 1) intention to breastfeed predicts breastfeeding 2) women intend to breastfeed when they evaluate it positively, when their important people recommend breastfeeding, and when they believe they can breastfeed without difficulty (19). Janke developed the breastfeeding attrition prediction tools (BAPT) based on TPB and showed that BAPT was a valid instrument to identify women at risk for early weaning (20). Results of a few studies confirmed that BAPT could identify women at higher risks of early breastfeeding discontinuation (21-24).

\section{Objectives}

The purpose of this study was to translate BAPT into Farsi, assess its content validity, and examine the validity and reliability of the Farsi version of BAPT to identify women at risk of EBF discontinuation, in a sample of women from northeast of Iran.

\section{Materials and Methods}

This longitudinal study was a part of a larger study, which aimed to examine the relationship between maternal psychological state, quality of life, and breastfeeding duration. It was started in May 2011 in Shahroud city located in northeast of Iran. The Research committee of Shahroud University of Medical Sciences approved this study (approval No. 9004). All the procedures followed were in accordance with the ethical standards of the Ethics Committee of the Shahroud University of Medical Sciences (Approval No. 900.02). We obtained permission to use the BAPT from the author.

There are 10 primary healthcare centers in Shahroud, where almost all pregnant women had registered to receive free prenatal care. Since pregnant women were accessible in healthcare centers, we used the convenience-sampling method. The inclusion criteria were gestational age $>28$ weeks, singleton pregnancy, ability to read and write, and no serious medical conditions that would prevent breastfeeding. We selected 390 consecutive women who attended ten urban healthcare centers affiliated to Shahroud University of Medical Sciences for prenatal care and met the inclusion criteria. Only 369 of them agreed to participate in the study over a period of six months. Eleven women who did not fill out the questionnaire completely were excluded from the study. Other exclusion criteria were fetal death, infant death before becoming four months old, and infant with major abnormalities or serious medical conditions which prevent breastfeeding. Only two infants died during the first two months of life and were excluded from the study. Finally, data from 356 women was collected. We included 11 women who were followed up to three months postpartum, but were not accessible at four months postpartum. According to the number of pregnant women registered at each center, we enrolled 30 to 40 pregnant women from each center. The sample size for this validation study was calculated based on the rule which indicates that a ratio of at least 5-10 subjects for each variable is adequate (25). Midwives at the primary healthcare centers were responsible for distributing and collecting questionnaires. They evaluated infant feeding method every month up to six months postpartum using face-to-face interview. According to WHO definitions, Infants receiving only breast milk with no additional foods or liquids were considered as receiving exclusive breastfeeding (3).

\subsection{Instruments}

\subsubsection{Socio-Demographic Questionnaire}

Women completed a questionnaire consisting of sociodemographic information, including age, education, family income, occupation, parity, and previous breastfeeding experience at the first visit. Intention to breastfeed was assessed by a question, using a six-point numerical rating scale $(1=$ definitely breastfeeding, 6 = definitely not breastfeeding).

\subsubsection{Infant Feeding Checklist}

The midwives assessed the Infant feeding practice every month up to six months postpartum by asking women to list all the fluids and foods that their infants received during the month before the interview, using a checklist.

\subsubsection{Breastfeeding Attrition Prediction Tools (BAPT)}

The BAPT developed by Janke is composed of 52 items divided into four subscales, including positive breastfeeding sentiment (PBS), negative breastfeeding sentiment (NBS), social and professional support (SPS), and perceived behavioral control (PBC) (20).

The 14-item PBS subscale consisted of positive attitudes about breastfeeding. A woman rated on a six-point scale (from completely disagree one point to completely agree 
six points) whether she agreed with the sentence. Then, the woman rated on a six-point Likert scale (from not important one point to very important six points) how important it was to her to use a feeding method with the given quality. Finally, we multiplied the scores for the attitude items with their corresponding importance and then added them up to create a score for positive breastfeeding attitudes, with higher scores indicating a more positive attitude toward breastfeeding.

The NBS subscale contained 15 items with negative meanings. The structure and scoring was the same as the PBS subscale, with higher scores indicating more negative attitudes toward breastfeeding.

The SPS subscale included 13 categories of important people. The woman rated on a six-point Likert scale (from definitely not breastfeeding one point to definitely breastfeeding six points) how these people thought she should feed her baby. Then, she rated on another sixpoint Likert scale (from not important one point to very important six points) to express how much she cared about these people's opinions. Finally, we multiplied the scores for the items on these two scales and then came up to create SPS score, with higher scores indicating greater support for breastfeeding.

The PBC subscale included 10 items. The woman marked the extent of their agreements with these statements on a six-point Likert scale (from completely disagree one point to completely agree six points), with higher scores implying that the woman had a stronger sense of perceived control over breastfeeding (20).

\subsection{Statistical Software}

We analyzed the data using SPSS version 12.0 (SPSS Inc., Chicago, IL, USA) for Windows. Stata version 12.0 was used for confirmatory factor analysis (CFA) and to plot the graph.

\subsection{Data Analysis}

To control the nonresponse bias, the individuals' mean imputation was used to deal with missing data. This method has been recommended as an appropriate and simple method (26). The total score of BAPT was calculated by summing up the scores of PBS, SPS, and BFC and subtracting the score of NBS. There was neither a ceiling nor a floor effect. We used descriptive statistics to describe the socio-demographic characteristics of the sample. We assessed reliability using the Cronbach's alpha internal validity coefficient and item-subscale correlation coefficients, alpha if the item deleted, and inter-item correlation coefficients. Alpha Cronbach values $>0.6$, itemsubscale correlation coefficients $>0.20$, and inter-item correlations coefficients $<0.80$ and higher than zero were regarded as acceptable. Alpha Cronbach value $<0.5$ was regarded as unacceptable. When an item-subscale correlation coefficient was $<0.2$, we removed the item, the deletion of which led to an increase of more than 0.1 in Alpha Cronbach coefficient of the subscale (27).

To determine the content validity, we calculated the content validity ratio (CVR) and content validity index (CVI). To determine the construct validity, we used both exploratory and confirmatory factor analyses. Exploratory factor analysis (EFA) was conducted utilizing principal component analysis with varimax rotation. The criterion for retaining the items was item loading $\geq 0.3$ (28). CFA was conducted by structural equation modeling. The method of estimation was principle components with varimax rotation. The input matrix was correlation matrix of data. We utilized four recommended indices (chisquare/df, root mean square error of approximation (RMSEA), comparative fit index (CFI), and standardized root mean square residual (SRMR)) (29). Relative chi-squares $<$ 5.00, a CFI value of $0.90<$, an RMSEA value of $<0.08$ and an SRMR value of $<0.08$ were considered as acceptable model fit (30).

Validity was further examined by known group comparison and the predictive validity. For the known group comparison, we used t-test to compare the mean score of the BAPT subscales in primiparous and multiparous women. For predictive validity, we used t-test to compare the mean score of the BAPT subscales in exclusive and nonexclusive breastfeeding women at four months postpartum.

\subsubsection{Process of Translation and Cultural Adaptation}

Two English language specialists translated the BAPT separately. Then, we discussed the differences between the two translated versions and created the final version. At the next step, a Ph.D. in English language who had not read the original version translated the Farsi version into English. Then, we sent this version to Janke for review. Finally, we made few minor revisions based on her comments.

\subsubsection{Content Validity}

The purpose of this step was to ensure that the items of the Farsi version of BAPT were essential, clear, simple and relevant to the Iranian culture. Both qualitative and quantitative methods were applied. In the qualitative phase, we invited an expert panel, consisted of 11 faculty members with different specialties, to evaluate and discuss the essentiality of the questionnaire items, its wording and scaling, and its relevancy to the Iranian culture. Seven experts argued that in the SPS subscale, three items were not relevant to our culture, e.g. La Leche League, a pediatrician, and a childbirth educator (although the breastfeeding promotion association is active in Iran, women are not aware of its activities or even its existence). In addition, two pediatricians argued that women did not consult with a pediatrician for breastfeeding in the antepartum period. Finally, midwives stated that there was no childbirth educator in the primary healthcare system where woman could receive free prenatal care. In the 
quantitative phase, experts expressed their ideas about essentiality of the items for the Iranian culture. We chose the Lawshe approach to determine the content validity ratio (CVR) (31). For assessing the necessity of the items, a three-point rating scale was used: a) not necessary, b) useful but not essential, and c) essential. We calculated a CVR for every item using the following formula;

$$
\mathrm{CVR}=\frac{\left[n-\left(\frac{N}{2}\right)\right]}{\frac{N}{2}}
$$

( $\mathrm{N}=$ the total number of experts and $\mathrm{n}=$ the number of experts who had chosen option c for each particular item). We computed a CVR for the total scale. According to the Lawshe table, an acceptable CVR value for 10 experts is 0.62. CVR of all the items of the instrument was 0.63 to 1, except for four items of SPS subscale which had a CVR $<0.62$. In the SPS subscale, three items were deleted; La Leche League, a pediatrician, and a childbirth educator. According to the experts' recommendations, we changed the fourth item from hospital nurse to health centers' personnel, since they regularly instructed women during pregnancy on how to breastfeed. Finally, the mean CVR for the total scale was 0.73 .

Afterwards, BAPT was given again to the experts to express their ideas about clarity, simplicity, and relevancy of each item in a four-point Likert scale (from a: not relevant, not simple, and not clear to d: very relevant, very simple, and very clear). The CVI for every item was calculated by dividing the total number of experts by the number of experts who had chosen c or d option for each particular item (e.g. a: not clear, b: needs major revision, c: clear but needs minor revision, d: clear). We calculated the CVI for relevancy, clarity, and simplicity of every item, according to the 11 experts' opinions on each item. Polit and Beck recommended 0.80 as the acceptable lower limit for the CVI value (32). CVI of all the items of the instrument were 0.8 to 1 and the mean CVI for the total scale was 0.89 .

\subsubsection{Pilot Study}

In the pilot study, we asked 20 low-educated pregnant women to fill out the instrument to examine the instructions for completing the scale. Most of the participants stated that they were confused and did not know what some items were asking. We revised the tool and converted the SPS subscale items into questions. In addition, NBS and PBS items which assessed the importance of every item for woman were converted into questions, without changing the meaning of the items. Then, we asked 10 pregnant women to fill out the revised translated BAPT and they could complete the instrument without difficulties.

\section{Results}

\subsection{Women's Characteristics}

The mean age of women was $26.17 \pm 4$.4. Eleven percent of the women had completed elementary school. All of the study participants were married. All the multiparous women had prior breastfeeding experience. Of the 345 participants, 80 (23.1\%) were on exclusive breastfeeding at four months postpartum. Women's characteristics are presented in Table 1.

\begin{tabular}{lc}
\hline Table 1. Participants' Characteristics ${ }^{\mathrm{a}}$ & \\
\hline Variables & Value \\
\hline Age, $\mathbf{y}$ & \\
$\quad \leq 20$ & $30(8.5)$ \\
\hline $20-29$ & $238(67.0)$ \\
$\quad \geq 30$ & $87(24.5)$ \\
\hline Education level, $\mathbf{y}$ & \\
\hline Primary, $<7$ & $43(12.0)$ \\
\hline High school, 7-12 & $214(60.3)$ \\
\hline University, $12<$ & $99(27.7)$ \\
\hline Family income, million Rialls & $4.24 \pm 1.50$ \\
\hline Infant birth weight, $\mathbf{g}$ & $3217 \pm 383$ \\
\hline Parity & \\
\hline Primiparous & $209(58.7)$ \\
\hline Multiparous & $147(43.3)$ \\
\hline Desirability of pregnancy & \\
\hline Wanted & $313(88)$ \\
\hline Unwanted & $43(12)$ \\
\hline Employment & $324(91)$ \\
\hline No & $32(9)$ \\
\hline Yes & \\
\hline Mode of delivery & $184(51.6)$ \\
\hline Vaginal delivery & \\
\hline a Data are presented as No.(\%) and mean \pm SD. \\
\hline
\end{tabular}

\subsection{Reliability}

We assessed the reliability of the Farsi version of BAPT by Cronbach's alpha coefficient and item-subscale correlations. The instrument overall Cronbach's alpha coefficient was 0.775. Subscale reliabilities ranged from 0.657 to 0.865 . Cronbach's alpha coefficients of individual subscales are presented in Table 2. The Cronbach's alpha was acceptable for all the subscales. The item-subscale analysis reflects the relationship between the value of each item of the subscale and the total value of the entire subscale. Item-subscale correlation coefficients of three items of the PBS scale (items No. 1, 5, and 17), one item of NBS (item No. 6), one item of SPS (the sixth item), and one item of BFC (the fifth item) were lower than 0.3. Cronbach's alpha coefficient of the scales improved slightly (< 0.01) if we delete these items. 
Mortazavi F et al.

Table 2. Descriptive Statistics and Alpha Cronbach for the Breastfeeding Attrition Prediction Tools BAPT Subscales

\begin{tabular}{|c|c|c|c|c|c|c|c|}
\hline \multirow[t]{2}{*}{ Subscale } & \multirow[t]{2}{*}{ Mean \pm SE } & \multirow[t]{2}{*}{ Median } & \multirow[t]{2}{*}{ Minimum } & \multirow[t]{2}{*}{ Maximum } & \multirow[t]{2}{*}{ All } & \multicolumn{2}{|c|}{ Alpha Cronbach } \\
\hline & & & & & & Primiparas & Multiparas \\
\hline PBS & $419.1 \pm 2.5$ & 422.5 & 90 & 504 & 0.657 & 0.677 & 0.631 \\
\hline NBS & $205.0 \pm 4.6$ & 202.5 & 51 & 462 & 0.857 & 0.853 & 0.863 \\
\hline SPS & $277.7 \pm 3.2$ & 288.0 & 60 & 360 & 0.767 & 0.729 & 0.809 \\
\hline BFC & $51.5 \pm 0.4$ & 52.0 & 16 & 60 & 0.865 & 0.852 & 0.874 \\
\hline Total $^{\mathrm{a}}$ & $547.8 \pm 7.1$ & 545.5 & 195 & 820 & 0.775 & 0.757 & 0.796 \\
\hline
\end{tabular}

a Total score = PBS + SPS + BFC - NBS; PBS, positive breastfeeding sentiment (14 items) (14-504); NBS, negative breastfeeding sentiment (15 items) (15 540); SPS, social and professional support (10 items) (10-360); BFC, breastfeeding control (10 items) (10 - 60); total score, 49 items (49-384).

Table 3. Results of Confirmatory Factor Analysis a

\begin{tabular}{lcccccc}
\hline & Chi-square & P Value & Chi-square/df & RMSEA & SRMR & CFI \\
\hline Farsi version of the BAPT & 2027 & $<0.001$ & 1.8 & 0.049 & 0.064 & 0.91 \\
Recommended value & - & $\mathrm{P}>0.05$ & $<5.00$ & $<0.08$ & $<0.08$ & $0.90<$ \\
\hline
\end{tabular}

a Abbreviations: Chi-square/df, minimum fit function/degree of freedom; RMSEA, root mean square error of approximation; SRMR, standardized root mean square residual; CFI, comparative fit index.

Table 4 . BAPT Subscales According to Women's Breastfeeding Experience ${ }^{\text {a, b }}$

\begin{tabular}{lcccc}
\hline BAPT Subscales & Without Breastfeeding Experience $(\mathbf{n}=\mathbf{2 2 8})$ & With Breastfeeding Experience $(\mathbf{n}=\mathbf{1 2 8})$ & T Value & P Value \\
\hline PBS & $417.0 \pm 48.5$ & $422.8 \pm 43.5$ & -1.1 & 0.278 \\
NBS & $206.5 \pm 85.0$ & $201.9 \pm 89.4$ & 0.4 & 0.613 \\
SPS & $280.3 \pm 56.3$ & $272.6 \pm 68.1$ & 1.1 & 0.27 \\
BFC & $49.9 \pm 7.2$ & $54.3 \pm 6.0$ & -5.8 & $<0.001$ \\
Total score & $541.2 \pm 117.1$ & $546.1 \pm 131.5$ & -0.5 & 0.723 \\
\hline
\end{tabular}

\footnotetext{
a Data are presented as mean \pm SD.

b Abbreviations: PBS, positive breastfeeding sentiment; NBS, negative breastfeeding sentiment; SPS, social and professional support; BFC, breastfeeding
} control.

\subsection{Construct Validity}

We evaluated the construct validity using exploratory and confirmatory factor analyses. We conducted EFA on 49 items. The Kaiser-Meyer-Olkin (KMO) coefficient was 0.822, which indicated that the sample size for the exploratory factor analysis was enough. Bartelet test of sphericity showed that chi-square was significant (chi-square $=5363$, $\mathrm{P}<0.001$ ), which meant that the levels of correlation between the items of instrument were enough to perform factor analysis on our sample. Principal component analysis with varimax rotation was conducted on 49 items. We instructed the software to extract four factors. The Farsi version of the BAPT included the same four factors that the original study found: 1 . negative breastfeeding sentiment, 2. breastfeeding control, 3. positive breastfeeding sentiment, 4. social and professional support. These factors altogether explained $35.27 \%$ of the variance. The first factor was NBS which included 15 items and had an eigenvalue $=$ 7.08 and explained $11.2 \%$ of the variance. The second factor was BFC with an eigenvalue $=4.86$ which explained $9.7 \%$ of the variance. The third factor was PBS with an eigenvalue $=$ 3.28 which explained 7.4\% and the last factor was SPS with a eigenvalue $=2.78$ which explained $6.9 \%$ of the variance. The results indicated that two items (PBS 1 and PBS 17) had an item loading $<0.3$. In addition, cross loading was observed for the two mentioned items and for BFC 5.

CFA was used to examine the factorial construct validity of the instrument. The covariance matrix of the 49 items was fitted with the apriori four-factor model in the CFA. Table 3 shows the results of CFA. Results of the CFA revealed the following factor loads: the NBS subscale from 0.27 to 0.74 , the BPC subscale from 0.31 to 0.79 , the PBS subscale from 0.23 to 0.65 , and the SPS subscale from 0.25 to 0.73 . Figure 1 illustrates a graphic of the instrument in a CFA model. The scale items are associated with their own subscale, define their own factors, and confirm the four-factor structure. 


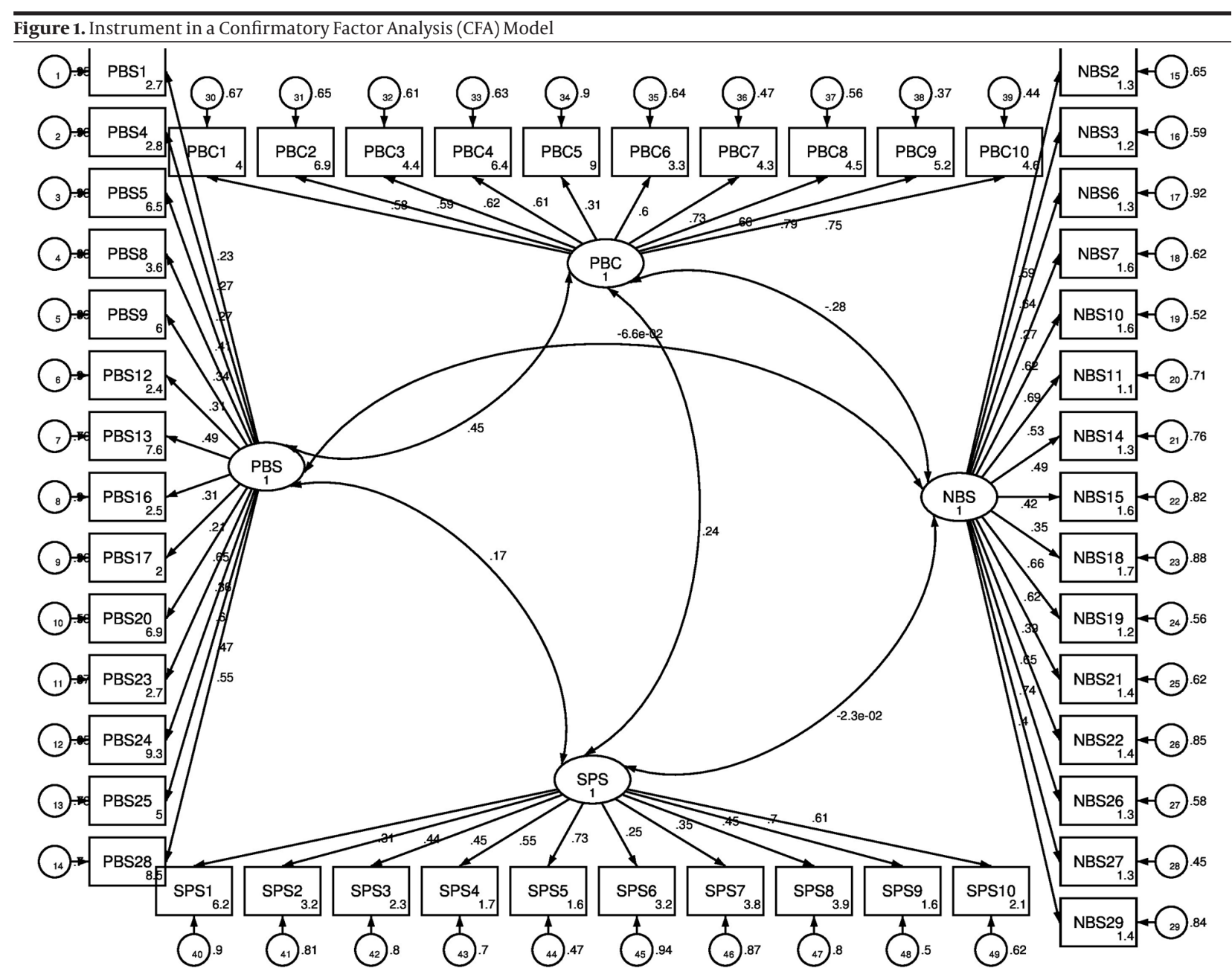

Abbreviations: PBS, positive breastfeeding sentiment; NBS, negative breastfeeding sentiment; SPS, social and professional support; PBC, perceived behavioral control.

\begin{tabular}{|c|c|c|c|c|}
\hline BAPT Subscales & Exclusive Breastfeeding $(\mathbf{n}=\mathbf{8 0})$ & Non-Exclusive Breastfeeding $(\mathrm{n}=\mathbf{2 6 5})$ & TValue & PValue \\
\hline PBS & $427.3 \pm 41.8$ & $416.2 \pm 48.1$ & 1.8 & 0.063 \\
\hline NBS & $192.1 \pm 85.8$ & $209.9 \pm 86.2$ & -1.6 & 0.106 \\
\hline SPS & $285.5 \pm 70.1$ & $283.2 \pm 57.1$ & 0.9 & 0.785 \\
\hline BFC & $53.1 \pm 6.2$ & $51.1 \pm 7.3$ & 2.3 & 0.019 \\
\hline Total score & $576.4 \pm 126.5$ & $537.8 \pm 121.0$ & 2.3 & 0.024 \\
\hline
\end{tabular}

${ }^{a}$ Abbreviations: PBS, positive breastfeeding sentiment; NBS, negative breastfeeding sentiment; SPS, social and professional support; BFC, breastfeeding control.

Table 6. Results of Multiple Regression Analysis of Antepartum Intention to Breastfeed on Mother's Attitude, Subjective Norms, and Control Over Breastfeeding ${ }^{\mathrm{a}}$

\begin{tabular}{lcccccc}
\hline \multirow{2}{*}{ Model } & \multicolumn{2}{c}{ Unstandardized Coefficients } & Standardized Coefficients & \multirow{2}{*}{ T Value } & \multirow{2}{*}{ P Value } \\
\cline { 2 - 3 } & B & SE & Beta & & 10.315 & $<0.001$ \\
Constant & 3.997 & 0.387 & 0.140 & 2.569 & 0.011 \\
PBS & 0.002 & 0.001 & -0.105 & -1.994 & 0.047 \\
NBS & -0.001 & $<0.001$ & 0.112 & 2.148 & 0.032 \\
SPS & 0.001 & 0.001 & 0.142 & 2.524 & 0.012 \\
BFC & 0.014 & 0.005 & &
\end{tabular}

a Abbreviations: PBS, positive breastfeeding sentiment; NBS, negative breastfeeding sentiment; SPS, social and professional support; BFC, breastfeeding control; B, Unstandardized Coefficient; SE, standard error. 


\subsection{Known Group Comparison}

A known group comparison method was adopted to provide support for the discriminant validity of the instrument. We assumed that pregnant women who had breastfeeding experience would have higher levels of BFC than women who had no previous breastfeeding experience. The results showed that the BFC subscale of the Farsi version of BAPT could discriminate women with and without breastfeeding experience $(\mathrm{P}<0.001)$ (Table 4).

\subsection{Predictive Validity}

We also evaluated validity by determining the predictive validity of the instrument. We assumed that women, who had positive attitudes, support, and perceived control about breastfeeding would have exclusively breastfeed their babies. The scores were compared by infant-feeding method at four months postpartum. We found that the total score of the BAPT and the score of the BFC subscale were higher in women who were on exclusive breastfeeding at four months postpartum than women who were not on exclusive breastfeeding $(\mathrm{P}<0.05)$ (Table 5). In addition, we found that all the four subscales of the Farsi version of BAPT could predict breastfeeding intension (Table 6).

\section{Discussion}

This study aimed to translate BAPT into a Farsi version while preserving the same ideas across the linguistic and cultural areas. To our knowledge, this is the first study in Iran to translate and validate BAPT. BAPT focuses on predicting a woman's likelihood to discontinue breastfeeding. In this study, we examined its reliability and validity with a new population, in which there was a high prevalence of breastfeeding continuation as well as low percentage of exclusive breastfeeding.

The assessment of internal consistency, measured by Cronbach's alpha, showed that all the alpha values were greater than 0.60. Our results showed that the instrument had reasonable reliability in Iranian culture, which was in agreement with results of other studies $(20,22$, 24). The item-subscale analysis showed that there were six item-subscale correlation coefficients lower than 0.3. It is recommended that items with correlation coefficients lower than 0.3 be removed from the instrument, provided that there would be an increase of more than 0.10 in the $\alpha$ coefficient when they were removed from the tool (33). In this study, there was an increase of less than 0.01 in Cronbach's alpha for these items, so we did not remove them from the tool.

The results of content validity showed that the items of the instrument were understandable and relevant to Iranian culture. We assessed construct validity using EFA and CFA. EFA could successfully detect the four factors, which altogether explained $35.27 \%$ of variance, which was lower than $50 \%$, while all other previous studies achieved it less than $42 \%(20,21,24)$. Two items (PBS1 and PBS 17) had item loadings $<0.3$. Cross loading was observed for three items (PBS 1, PBS 17, and BFC 5). We included these items for further evaluation in future studies. These items were: "breastfeeding is more convenient than formula feeding (PBS 1)", "breastfeeding is less costly than formula feeding (PBS 17)", and "I am determined to breastfeeding (BFC 5)". The factor loadings of the first two items were bigger than the NBS factor. It is possible that these two items had low correlations with the PBS because most women from Shahroud believed that breastfeeding was not more economical or convenient than formula feeding or they did not care about the cost and convenience. However, they follow Islamic tradition, which recommends that women should breastfeed their babies up to two years of age (34). Consistent with the results of Muslu's study, the CFA results showed that the model was in accordance with the proposed four-structure model (24).

Comparison of women with and without previous breastfeeding experience revealed that experienced women had a higher control score. This finding, which provided evidence for predictive validity, was consistent with previous researches $(24,35)$. The comparison of women who continued breastfeeding exclusively with women who did not continue breastfeeding exclusively showed that the BFC subscale could discriminate between the two groups. In Muslu's study, the mean scores of PBS and SPS subscales were higher in breastfeeding group than bottle feeding group (24). In Dick's study, modified BAPT was a predictor of breastfeeding discontinuation before eight weeks (21). In addition, the instrument could predict the intension severity of breastfeeding. These results were consistent with Wambach's findings, which indicated that attitude and control were predictors of breastfeeding intention (36). Lewallen revised BAPT and found that it could differentiate between women who planned to breastfeed and those who planned to formula feed (37). Gill also modified BAPT and showed that it was a good measure of intention to breastfeed (22). In Swanson's study on adolescents, breastfeeding knowledge and subjective norms were important predictors of positive breastfeeding beliefs and future intentions to breastfeed (38).

We adapted the original English version of BAPT to Farsi. The results of this study showed that the Farsi version of BAPT was similar to the original version. We recommend that further studies in Iran be conducted to examine the Farsi version of BAPT to identify women at higher risk of breastfeeding discontinuation where it is more prevalent.

\subsection{Limitations and Strength}

There were some limitations regarding the study. First, the number of items in the scale were high (88) and women had to read and mark several demographic items as well. This might have caused women to answer the ques- 
tions with low accuracy and precision. However, we asked women to answer the questionnaire at home to lower the number of missed items and the probability of misunderstanding due to possible fatigue. Furthermore, we asked the women to check the questionnaire again when they wanted to provide it back to the midwife. Filling out the questionnaire at home is an acceptable method of data gathering; however, it is not possible to control the situation at which the respondents complete the questionnaire. The strength of the study was that it covered all the pregnant women who were registered throughout healthcare centers of Shahroud, which increased the external validity of the results.

\section{Acknowledgements}

The authors wish to thank all the midwives and women who participated in the study. This work was part of a Ph.D. dissertation titled "assessment of breastfeeding attrition prediction tools in prediction of breastfeeding initiation and continuation".

\section{Authors' Contributions}

All the authors contributed significantly. Forough Mortazavi was the main investigator and wrote the proposal, collected the data, and revised the final draft. Seyed Abbass Mousavi contributed to the study design and first draft writing. Reza Chaman contributed to the interpretation of the findings and first draft writing. Ahmad Khosravi contributed to the statistical analysis and first draft writing. Jill $\mathrm{R}$ Janke was the creator of BAPT and contributed to the comparison of the back translation of the instrument Farsi version with the original English version. The correspondent Author was Dr. Seyed Abbass Mousavi.

\section{Funding/Support}

This study was partially financed by Shahroud University of Medical Sciences (approval No. 9004).

\section{References}

1. Kramer MS, Kakuma R. Optimal duration of exclusive breastfeeding. Cochrane Database Syst Rev. 2002(1):CD0 03517.

2. Howel D, Ball H. Association between length of exclusive breastfeeding and subsequent breastfeeding continuation.J Hum Lact. 2013;29(4):579-85.

3. W.H.O. The Global strategy for infant and young child feeding Geneva: 2002.

4. Marandi A, Afzali HM, Hossaini AF. The reasons for early weaning among mothers in Teheran. Bull World Health Organ. 1993;71(5):561-9.

5. Olang B, Farivar K, Heidarzadeh A, Strandvik B, Yngve A. Breastfeeding in Iran: prevalence, duration and current recommendations. Int Breastfeed J. 2009;4:8.

6. UNICEF [27 Dec 2013];Iran, Islamic Republic of - Statistics. 2011 avilable from: \{ http://www.unicef.org/infobycountry/iran_statistics.html

7. Veghari G, Mansourian A, Abdollahi A. Breastfeeding status and some related factors in northern iran. Oman Med J.2011;26(5):3428.
8. Lou Z, Zeng G, Huang L, Wang Y, Zhou L, Kavanagh KF. Maternal reported indicators and causes of insufficient milk supply.J Hum Lact. 2014;30(4):466-73.

9. Blyth R, Creedy DK, Dennis CL, Moyle W, Pratt J, De Vries SM. Effect of maternal confidence on breastfeeding duration: an application of breastfeeding self-efficacy theory. Birth. 2002;29(4):27884.

10. Oliver-Roig A, d'Anglade-Gonzalez ML, Garcia-Garcia B, Silva-Tubio JR, Richart-Martinez M, Dennis CL. The Spanish version of the Breastfeeding Self-Efficacy Scale-Short Form: reliability and validity assessment. Int J Nurs Stud. 2012;49(2):169-73.

11. Arts M, Geelhoed D, De Schacht C, Prosser W, Alons C, Pedro A. Knowledge, beliefs, and practices regarding exclusive breastfeeding of infants younger than 6 months in Mozambique: a qualitative study. J Hum Lact. 2011;27(1):25-32.

12. Gilmour C, Hall H, McIntyre M, Gillies L, Harrison B. Factors associated with early breastfeeding cessation in Frankston, Victoria: a descriptive study. Breastfeed Rev. 2009;17(2):13-9.

13. Mueffelmann RE, Racine EF, Warren-Findlow J, Coffman MJ. Perceived Infant Feeding Preferences of Significant Family Members and Mother's Intention to Exclusively Breastfeed. J Hum Lact. 2014.

14. Haroon S, Das JK, Salam RA, Imdad A, Bhutta ZA. Breastfeeding promotion interventions and breastfeeding practices: a systematic review. BMC Public Health. 2013;13 Suppl 3:S20.

15. Ahmad MO, Sughra U, Kalsoom U, Imran M, Hadi U. Effect of antenatal counselling on exclusive breastfeeding. J Ayub Med Coll Abbottabad. 2012;24(2):116-9.

16. Dodgson JE, Henly SJ, Duckett L, Tarrant M. Theory of planned behavior-based models for breastfeeding duration among Hong Kong mothers. Nurs Res. 2003;52(3):148-58.

17. Giles M, Connor S, McClenahan C, Mallett J, Stewart-Knox B, Wright M. Measuring young people's attitudes to breastfeeding using the Theory of Planned Behaviour. J Public Health (Oxf). 2007;29(1):17-26.

18. McMillan B, Conner M, Woolridge M, Dyson L, Green J, Renfrew $\mathrm{M}$, et al. Predicting breastfeeding in women living in areas of economic hardship: Explanatory role of the theory of planned behaviour. Psychol Health. 2008;23(7):767-88.

19. Ajzen I. The theory of planned behavior. Organ Behav Hum Decis Process. 1991;50(2):179-211.

20. Janke JR. Development of the Breast-Feeding Attrition Prediction Tool. Nurs Res. 1994;43(2):100-4.

21. Dick MJ, Evans ML, Arthurs JB, Barnes JK, Caldwell RS, Hutchins SS, et al. Predicting early breastfeeding attrition. J Hum Lact. 2002;18(1):21-8.

22. Gill SL, Reifsnider E, Lucke JF, Mann AR. Predicting breast-feeding attrition: adapting the breast-feeding attrition prediction tool. $J$ Perinat Neonatal Nurs. 2007;21(3):216-24.

23. Evans ML, Dick MJ, Lewallen LP, Jeffrey C. Modified breastfeeding attrition prediction tool:prenatal and postpartum tests.J Perinat Educ. 2004;13(1):1-8.

24. Karayagiz Muslu G, Basbakkal Z, Janke J. The Turkish version of the breastfeeding attrition prediction tool. J Hum Lact. 2011;27(4):350-7.

25. Tinsley HE, Tinsley DJ. Uses of factor analysis in counseling psychology research. J Couns Psychol.1987;34(4):414.

26. Shrive FM, Stuart H, Quan H, Ghali WA. Dealing with missing data in a multi-question depression scale: a comparison of imputation methods. BMC Med Res Methodol. 2006;6:57.

27. Streiner DL. Starting at the beginning: an introduction to coefficient alpha and internal consistency. J Pers Assess. 2003;80(1):99103.

28. Dr. Carolyn Waltz PhD RNF, Dr. Ora Lea Strickland PhD RNF, Dr Elizabeth Lenz PhD RNF. Measurement in Nursing and Health Research: Fourth Edition.: Springer Publishing Company; 2010.

29. Kline RB. Principles and Practice of Structural Equation Modeling. Guilford Press; 2005.

30. Bentler PM, Bonett DG. Significance tests and goodness of fit in the analysis of covariance structures. Psychol Bull.1980;88(3):588

31. Lawshe $\mathrm{CH}$. A quantitative approach to content validity. Pers Psychol.1975;28(4):563-75. 


\section{Mortazavi F et al.}

32. Polit DF, Beck CT, Owen SV. Is the CVI an acceptable indicator of content validity? Appraisal and recommendations. Res Nurs Health. 2007;30(4):459-67.

33. Schuttert JB, Liu MH, Gliem N, Fiedler GM, Zopf S, Mayer C, et al. Human renal fibroblasts derived from normal and fibrotic kidneys show differences in increase of extracellular matrix synthesis and cell proliferation upon angiotensin II exposure. Pflugers Arch. 2003;446(3):387-93.

34. Torkzahrani S. Commentary: childbirth education in iran.J Perinat Educ. 2008;17(3):51-4.

35. Wells KJ, Thompson NJ, Kloeblen-Tarver AS. Development and psychometric testing of the prenatal breast-feeding self-efficacy scale. Am J Health Behav. 2006;30(2):177-87.

36. Wambach KA. Breastfeeding intention and outcome: a test of the theory of planned behavior. Res Nurs Health. 1997;20(1):51-9.

37. Lewallen LP, Dick MJ, Wall Y, Zickefoose KT, Hannah SH, Flower J, et al. Toward a clinically useful method of predicting early breast-feeding attrition. Appl Nurs Res. 2006;19(3):144-8.

38. Swanson V, Power K, Kaur B, Carter H, Shepherd K. The impact of knowledge and social influences on adolescents' breast-feeding beliefs and intentions. Public Health Nutr. 2006;9(3):297-305. 